\title{
Choose change: Situation modification, distraction, and reappraisal in mild versus intense negative situations
}

\author{
Bram Van Bockstaele ${ }^{1,2}$ (D) Ludovica Atticciati $^{1} \cdot$ Anu P. Hiekkaranta $^{1,3} \cdot$ Helle Larsen $^{1} \cdot$ Bruno Verschuere $^{1}$
}

Published online: 19 October 2019

(c) The Author(s) 2019

\begin{abstract}
Despite the theoretical importance and applied potential of situation modification as an emotion regulation strategy, empirical research on how people change situations to regulate their emotions is scarce. Meanwhile, existing paradigms typically allowed participants to avoid the entire situation, thus confounding situation modification with situation selection. In our current experiments, participants could choose between partially modifying their negative emotional environment without avoiding it entirely and two well-established emotion regulation strategies (reappraisal and distraction). Participants did choose situation modification (Experiments 1-2) and they did so more often for intense than for mild stimuli in Experiment 2. In addition, modifying the stimulus display effectively helped downregulating negative affect (Experiments 1-2). Finally, in both experiments, participants opted more for distraction for intense compared to mild stimuli, while they opted more for reappraisal for mild compared to intense stimuli. Presenting a first step in developing a paradigm that allows people to exert control over but to not avoid emotion-provoking situations, we thus show that changing one's environment helps regulating one's emotions. More generally, our findings indicate that people prefer to regulate their emotions using disengagement strategies (situation modification and distraction) with high-intensity relative to low-intensity negative situations, while they prefer engagement strategies (reappraisal) with low-intensity relative to high-intensity negative situations.
\end{abstract}

Keywords Emotion regulation $\cdot$ Situation modification $\cdot$ Reappraisal $\cdot$ Distraction

Electronic supplementary material The online version of this article (https://doi.org/10.1007/s11031-019-09811-8) contains supplementary material, which is available to authorized users.

Bram Van Bockstaele

B.D.Vanbockstaele@uva.nl;

Bram.Vanbockstaele@gmail.com

Ludovica Atticciati

Ludovica.Atticciati@gmail.com

Anu P. Hiekkaranta

Anupauliina.Hiekkaranta@kuleuven.be

Helle Larsen

H.Larsen@uva.nl

Bruno Verschuere

B.J.Verschuere@uva.nl

1 Department of Psychology, University of Amsterdam, Nieuwe Achtergracht 129-B, 1018 WT Amsterdam, The Netherlands

2 Research Institute of Child Development and Education, University of Amsterdam, Amsterdam, The Netherlands

3 Present Address: Department of Neurosciences, Katholieke Universiteit Leuven, Leuven, Belgium
When we have a quick beer to settle our nerves right before a first date, when we interpret a lapse in conversation as a sign that we are both just enjoying our meals, or when we binge-watch our favourite television show to avoid thinking about why he or she does not return our calls: In all these occasions, we are regulating our emotions. Emotion regulation is commonly defined as "the processes by which individuals influence which emotions they have, when they have them, and how they experience and express these emotions" (Gross, 1998, p. 275). According to Gross' (1998, 2015) influential process model of emotion regulation, emotions can be regulated by selecting or avoiding entire emotionevoking situations (situation selection), by altering aspects of physical situations in order to change their emotional impact (situation modification), by guiding our attention to specific aspects of situations (attention deployment), by changing its meaning (cognitive change), or by influencing the emotional response itself (response modulation).

There is now an abundance of research supporting the idea that people effectively use attention deployment, cognitive change, and response modulation to regulate emotions 
(Webb et al. 2012a). However, studies on how people use situation selection and situation modification to regulate their emotions are surprisingly rare (Gross 2015). The need for experimental work on situation modification is all the more pressing because commonly considered effective and adaptive strategies, like reappraisal, may only be effective in situations that cannot be controlled. Indeed, while higher reappraisal ability has been related to less depressive symptoms in people who were confronted with uncontrollable stress, higher reappraisal ability was associated with more depressive symptoms in people who were confronted with controllable stress (Troy et al. 2013). In a similar vein, it has been shown that greater well-being is associated with more frequent reappraisal use in uncontrollable situations but with less frequent reappraisal use in controllable situations (Haines et al. 2016). For situations that can be changed, it may thus be more adaptive to modify rather than to reappraise them. Hence, experimental research on how modifying one's situation helps regulating one's emotions is imperative.

To the best of our knowledge, there are two studies on emotion regulation through situation modification. Vujovic et al. (2014) presented neutral and negative pictures, and allowed participants to try to erase these pictures by pressing the spacebar. They found that participants attempted to erase high-arousing negative pictures more often than low-arousing negative pictures. This paradigm, however, did not differentiate between situation selection and situation modification: Physically avoiding an emotion-evoking situation creates an entirely new situation and therefore is more akin to situation selection than to situation modification. Livingstone and Isaacowitz (2015) offered participants a range of positive, negative, and neutral stimuli that they could freely interact with in a situation selection block, and an option to skip sections of positive and negative videos in a situation modification block. Although participants chose to skip certain parts of videos, again, choosing to skip sections of videos terminates the presentation of the emotionevoking stimulus and therefore qualifies as avoidance rather than situation modification. Situation modification involves changing aspects of a physical situation to change its emotional impact, without simply avoiding the situation. Isolating situation modification from avoidance is crucial because avoidance decreases exposure to challenging situations, thus hampering learning and more long-term adaptation, and is related to more psychopathology, while situation modification (or problem-solving) has been related to less psychopathology (Aldao et al. 2010).

In the present study, we aimed to add to the literature on emotion regulation through situation modification by isolating situation modification from situation selection. While our participants were given the option to change a part of the stimulus display, they could not avoid the situation entirely.
More specifically, we presented participants with two negative pictures. Upon choosing situation modification, only one of the pictures faded gradually without being erased completely. In real life, we consider this equivalent to lowering the volume when watching a scary movie, without changing the channel or even switching off the television. By presenting two stimuli and only partially changing one of them, our participants could not avoid the negative situation by ending the stimulus presentation. Instead, they only partially reduced the visual intensity of one aspect of the negative situation.

Given the paucity of research on emotion regulation through situation modification, our main research question was whether and how frequently participants would choose to use our operationalization of situation modification to downregulate negative emotions. To do so, we incorporated situation modification in the emotion regulation choice paradigm. This paradigm was developed in the light of recent accounts of emotion regulation stressing the importance of flexibly switching between emotion regulation strategies (e.g., Aldao et al. 2015; Bonanno and Burton 2013; Sheppes et al. 2011), which is for instance also reflected in Gross' (2015) extended process model and the action control perspective of Webb et al. (2012b). According to these views, adaptive emotion regulation is not determined by consistently using a specific "adaptive" strategy, but rather by the ability to determine the need for regulation, flexibly selecting an appropriate strategy to do so given both individual and situational constraints, and finally using the optimal strategy. The studies of Haines et al. (2016) and Troy et al. (2013) illustrate this point, showing that using reappraisal is only adaptive in situations that cannot be changed. To experimentally address emotion regulation strategy choice, Sheppes et al. (2011) developed a paradigm in which participants indicated how they wanted to downregulate negative emotions evoked by negative pictures, allowing the choice between distraction and reappraisal. Further illustrating that emotion regulation strategy preference depends on situational factors, participants consistently preferred reappraisal over distraction when the pictures were mildly negative, while they preferred distraction over reappraisal for highly negative pictures (see e.g., also Sheppes et al. 2014). More generally, these findings suggest that in highly negative situations, people prefer to disengage from emotional processing by emotionally blocking this information early on, before it gathers force. For less intense negative situations, people are more likely to invest cognitive resources in engaging with the incoming emotional information, thus modulating the emotional response.

Building on these findings, we incorporated situation modification in the emotion regulation choice paradigm by offering participants the option to modify the pictures on display in addition to the options to use distraction or 
reappraisal. Next to our main research question whether and how frequently participants would use situation modification, we also investigated the effects of stimulus intensity on strategy preferences. Following the findings of Sheppes et al. $(2011,2014)$, we expected participants to be more likely to use disengagement (i.e., situation modification and distraction) rather than engagement strategies (i.e., reappraisal) for emotionally intense stimuli, while we expected reappraisal to be preferred relative to distraction and situation modification for low-intensity scenes. As a secondary outcome, we also measured self-reported emotion regulation effectiveness. Mirroring our predictions for the choice data, we expected situation modification and distraction to be especially effective in high-intensity situations, while we expected reappraisal to be more effective in downregulating emotions in low-intensity situations.

Finally, we also addressed three exploratory questions. First, because we included the option to partially modify the stimulus display, trials in our paradigm could be considered as controllable stressors. In line with Troy et al. (2013, see also Haines et al. 2016), who found that more reappraisal in controllable situations was associated with higher levels of depression, we expected that more reappraisal use in response to our controllable stimulus displays would also be related to higher depression scores. Second, we addressed whether observed strategy preferences in the lab mirrored self-reported strategy preferences in real life, as measured by an emotion regulation strategy questionnaire. Third, it is typically assumed that reappraisal requires a lot of cognitive control, illustrated by the finding that lower levels of cognitive control affect reappraisal ability (Opitz et al. 2012). It has also been hypothesized that people with lower levels of cognitive control will be more likely to use situation modification than reappraisal (Urry and Gross 2010). We therefore explored whether observed strategy preferences were associated with a measure of cognitive control, expecting a positive relation between cognitive control and reappraisal preferences as well as a negative relation between cognitive control and situation modification preferences.

\section{Experiment 1}

\section{Method}

\section{Participants}

Thirty-eight unselected students of the University of Amsterdam (29 women, $M_{\text {age }}=26.7, S D=10.5$, range $=19-60$ ) participated in exchange for either course credits or $€ 10$. We based our sample size on the within-subjects effect of stimulus intensity on strategy choices in the Experiments of Sheppes et al. (2011), who found very large effects $\left(\eta_{\mathrm{p}}^{2}\right.$ reported by Sheppes et al. transformed to $f:$ all $f \mathrm{~s} \geq 0.86$ with samples of 16-20 participants; according to Cohen's (1992) guidelines, values for $f$ from 0.10 represent small effects, values from 0.25 represent medium effects, and values from 0.40 represent large effects). Anticipating a medium sized correlation of .30 between repeated measurements, we used G*Power (Faul et al. 2007) to determine that a sample of 20 participants would be enough to find large within-subjects effects $(f \geq 0.40)$ with a power of .80 in a MANOVA, while a sample of 46 participants would be needed to also detect medium sized effects $(0.25 \leq f \leq 0.40)$. Our chosen sample size of 38 participants was thus large enough to detect medium to large effects, with $f \geq 0.28$.

\section{Materials}

Emotional pictures were selected from the International Affective Picture System (IAPS; Lang et al. 2008). We created 26 pairs of low-intensity negative pictures (IAPS normative ratings: Mean valence $=3.36$, Mean arousal $=5.20$ ) and 26 pairs of high-intensity negative pictures (IAPS normative ratings: Mean valence $=1.80$, Mean arousal $=6.34$ ). We created the pairs so that the two pictures would fit together in terms of content as much as possible (e.g., two pictures of car crashes, two pictures of maltreated dogs, etc. Full lists of picture pairs are presented in the online Supplemental Materials, section Methods Experiment 1).

\section{Emotion regulation choice task}

Each trial of the emotion regulation choice task consisted of 5 chronological steps: Preview, preview rating, strategy choice, downregulation, and downregulation rating (Fig. 1). In the preview phase, a white fixation cross was presented for $1000 \mathrm{~ms}$ on a black screen, followed by a pair of pictures. Participants were asked to only look at these pictures and to judge which emotions they felt in response to the pictures. After $3000 \mathrm{~ms}$, the pictures were erased. Next, in the preview rating phase, participants indicated on 4 separate 9-point Likert scales how intensely they had experienced fear, disgust, sadness, and anger in response to the pictures $(1=n o t$ intense at all, $3=$ little intense, $7=$ fairly intense, $9=$ very intense). In the strategy choice phase, participants were asked to choose an emotion regulation strategy to downregulate their negative emotions. To do so, we presented a choice screen consisting of a short instruction sentence and three strategy buttons, labelled "Modify", "Focus attention", and "Reinterpret". Participants selected their chosen strategy by clicking with the mouse on the corresponding strategy button.

In the downregulation phase, we asked participants to downregulate the negative emotions they felt in response to the pictures. First, the same picture pair as in the preview 


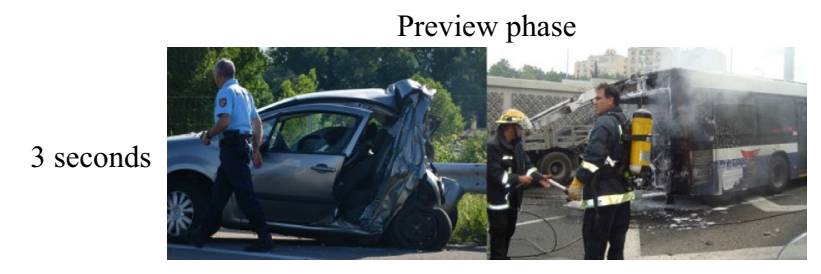

Preview rating phase

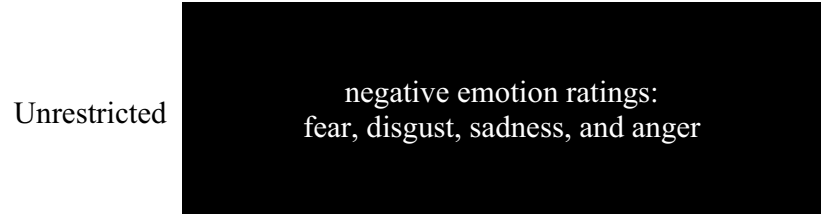

Strategy choice phase

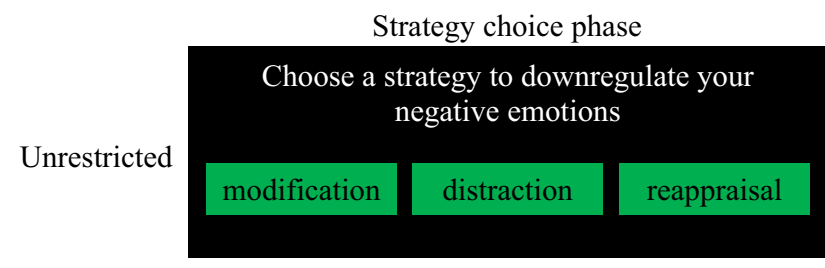

Downregulation phase

10 seconds

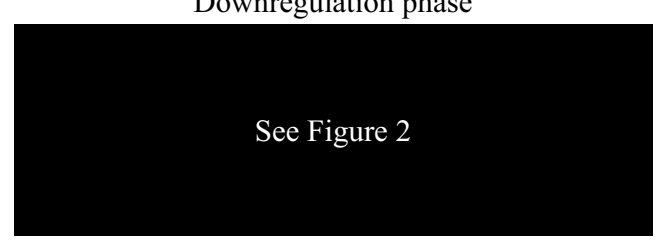

Downregulation rating phase

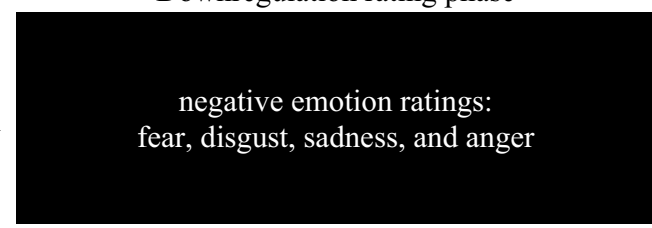

Fig. 1 Schematic overview of the emotion regulation choice paradigm. In compliance with copyright laws, pictures are similar but not identical to the ones used in the experiment

phase was presented on the screen. After $1000 \mathrm{~ms}$, the stimulus display changed in accordance with the strategy that participants had chosen. If participants had chosen to modify the situation (Fig. 2a), one of the two pictures faded to white by adding increasingly more opaque layers on top of the picture (opacity: $25 \%, 40 \%, 60 \%, 80 \%, 90 \%$ ). The opacity level was increased every $500 \mathrm{~ms}$. The other picture remained visible and the opacity of the faded picture did not reach $100 \%$ so participants could not completely avoid the emotional content. If participants had chosen to focus their attention (Fig. 2b), attention was guided to a neutral part of the picture pair by overlaying a white, semi-transparent layer on the rest of the picture pair, creating a spotlight similar to the procedure developed by Urry (2010). Importantly, this layer did not prevent seeing the details of the image; it only guided participants' attention to an emotionally neutral part of the stimulus display. If participants had chosen to reinterpret the stimulus display (Fig. 2c), a reappraisal sentence was presented underneath the pictures. These reappraisal sentences were inspired by Sheppes et al. (2014), and were compatible with both pictures in the stimulus display. All trials in the downregulation phase lasted for a total of $10,000 \mathrm{~ms}$, regardless of the strategy that participants had chosen. Finally, in the downregulation rating phase, participants again rated the intensity of fear, disgust, sadness, and anger that they had experienced during the downregulation phase.

The emotion regulation choice task consisted of 52 trials in total, divided over two blocks of 26 trials, separated by a self-paced break in between. Each block contained 13 high and 13 low-intensity picture pairs. Picture pairs were selected in a random order, and every picture pair was used in only one trial. Prior to the actual task, the emotion regulation strategies were explained in written instructions (see Supplemental Materials, section Methods Experiment 1). The choice procedure was explained and practiced in a total of six example trials with relatively mild negative pictures that were selected from the internet. In these example trials, participants were forced to pick each of the three strategies twice, to make sure that each of the three strategies was clear and that they knew what to expect with every choice. After the last practice trial and before starting the actual test blocks, the experimenter double-checked whether the procedure and the three different strategies were clear to the participant and provided them with extra information when needed.

\section{Questionnaires}

Cognitive emotion regulation questionnaire (CERQ) The CERQ (Garnefski et al. 2001) was used to assess selfreported use of emotion regulation strategies in real life. The scale consists of 36 items divided over 9 subscales of 4 items each. Each item is scored on a 4-point Likert scale. We focused on the subscales Positive refocusing (hypothesized to relate to distraction preferences), Refocus on planning (hypothesized to relate to situation modification preferences), and Positive reappraisal and Putting into perspective (hypothesized to relate to reappraisal preferences) (all Cronbach's alphas between .78 and .90).

Beck depression inventory (BDI-II) The Dutch translation of the BDI-II (Beck et al. 1996; van der Does 2002) was used to assess depressive symptoms. This questionnaire consists of 21 items, each scored on a 4-point Likert scale (Cronbach's alpha $=.92$ ).

\section{Flanker task}

A variant of the classical Eriksen flanker task (Eriksen and Eriksen 1974) was used as a measure of cognitive control. 


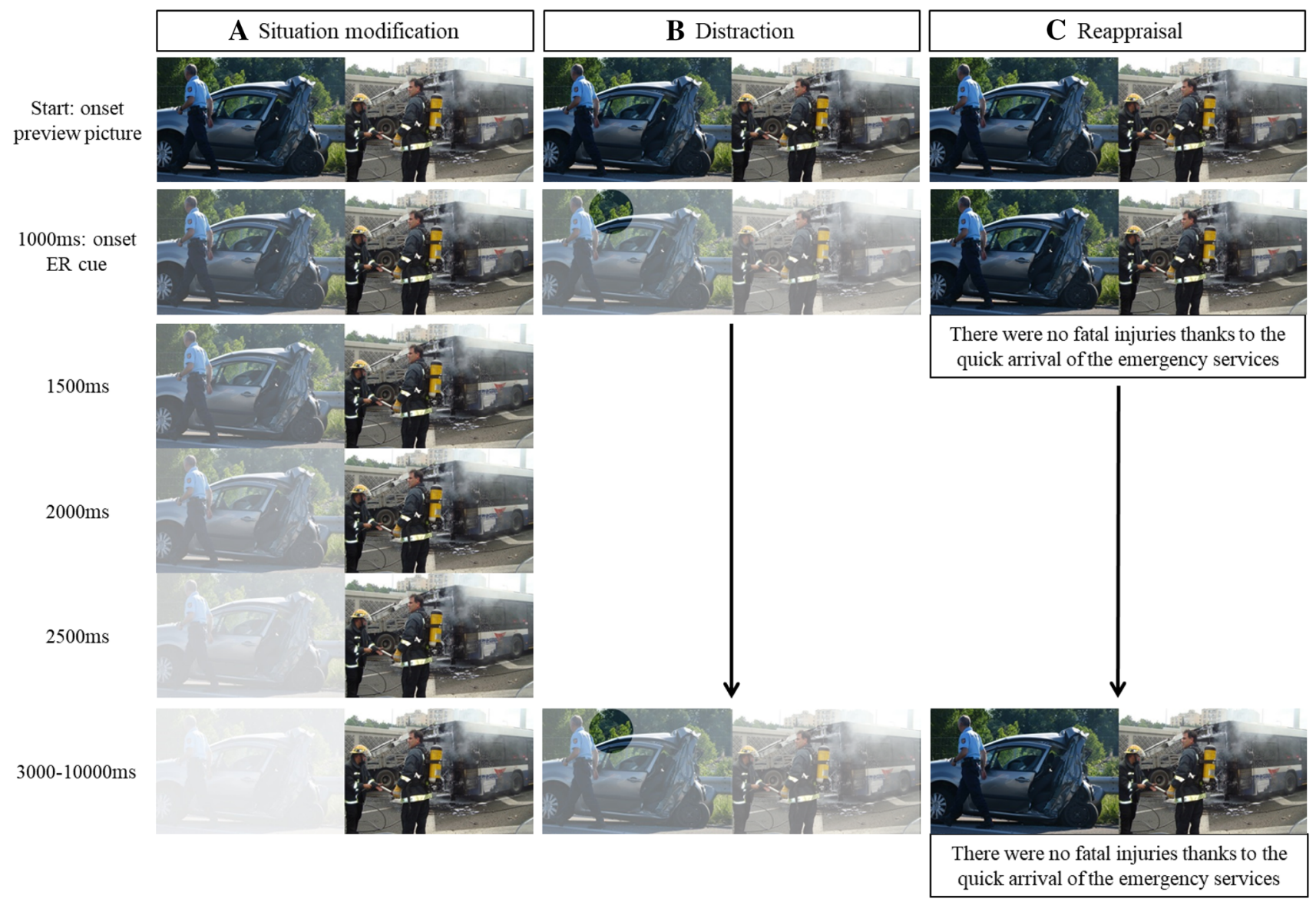

Fig. 2 Schematic overview of stimulus presentation sequences in the downregulation phase of Experiment 1

Each trial started with the presentation of a white fixation cross on a black background. After $500 \mathrm{~ms}$, the fixation cross was replaced by an arrow configuration consisting of five arrows with the central and flanking arrows either pointing in the same direction (congruent trials: " $>>>>>$ " or " $<<<<<$ ") or in opposite directions (incongruent trials: " $>><>>$ " or " $<<><<$ "). Participants were asked to respond as quickly and as accurately as possible to the direction of the middle arrow by pressing either the left or the right arrow key of the keyboard. Trials ended as soon as a response was registered, and the inter-trial interval was $200 \mathrm{~ms}$. The test block consisted of 60 trials, with all possible arrow configurations presented 15 times. Prior to the test block, participants completed a practice block consisting of 12 trials (each arrow configuration 3 times) with feedback on incorrect responses.

\section{Procedure}

Participants were tested in individual sound-proof cubicles. Prior to the start of the experiment, participants were informed about the nature of the stimulus materials and those with blood/injury phobia were discouraged from participating because many of the pictures contained bloody scenes. After providing written informed consent, participants filled out the questionnaires (for details of exploratory questionnaires that were included in the study, see Supplemental Materials, section Methods Experiment 1), followed by the flanker task and the emotion regulation choice task. The entire procedure lasted for $1 \mathrm{~h}$ and was approved by the ethical committee of the University of Amsterdam (Ref. Number 2017-DP-7853).

\section{Results}

\section{Data reduction and scoring}

To address whether and how often participants used each strategy, we calculated strategy choice frequencies for highand low-intensity trials separately, as well as overall strategy choice frequencies (i.e., irrespective of stimulus intensity). Because the strategy choice data are compositional, we calculated log-transformed relative strategy preferences 
Fig. 3 Summary of emotion regulation results of Experiment 1, separating high and low-intensity stimuli. a Shows raw strategy choice percentages, separately for each strategy. b Shows relative strategy preferences for each of the three strategy comparisons. Values represent the log-transformed preference of the first-named strategy in each pair relative to the second-named strategy in each pair. Positive values thus reflect a preference for the first-named strategy in each comparison and negative values reflect a preference for the second-named strategy in each comparison. Inverting these values-i.e., calculating $\operatorname{EXP}(\mathrm{x})$-yields the untransformed preferences (e.g., the value of around 1.1 in the comparison between reappraisal and situation modification on low intensity trials indicates that reappraisal was chosen about $\operatorname{EXP}(1.1)=3$ times more often than situation modification). c Shows the selfreported emotion downregulation effect (i.e., preview ratings minus downregulation ratings), separately for each strategy. Large positive values thus indicate more downregulation. Error bars reflect standard errors of the mean in all panels

\section{A Strategy choice frequencies}

$\square$ Low Intensity $\square$ High Intensity

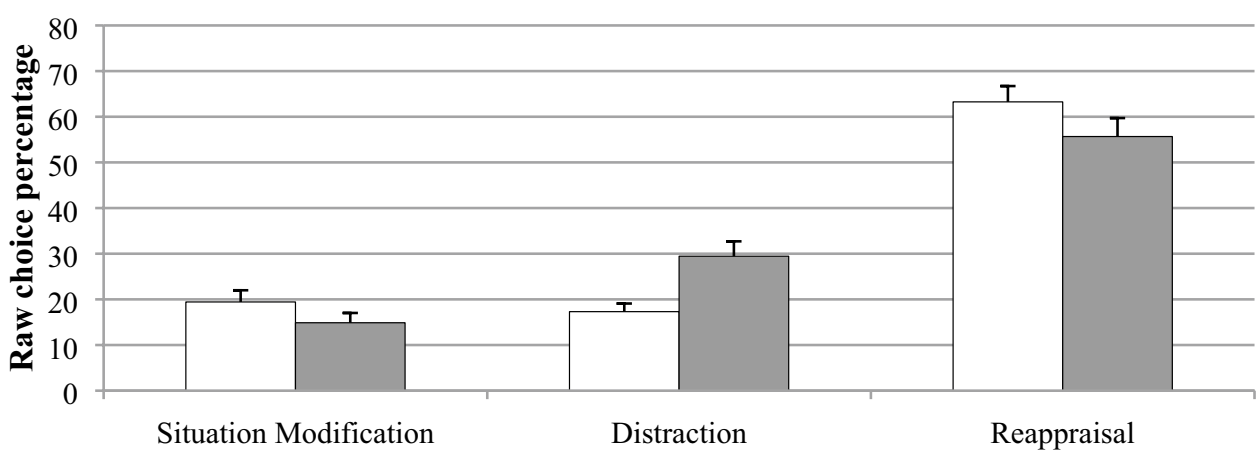

Emotion regulation strategy choice

\section{B Relative strategy preferences}

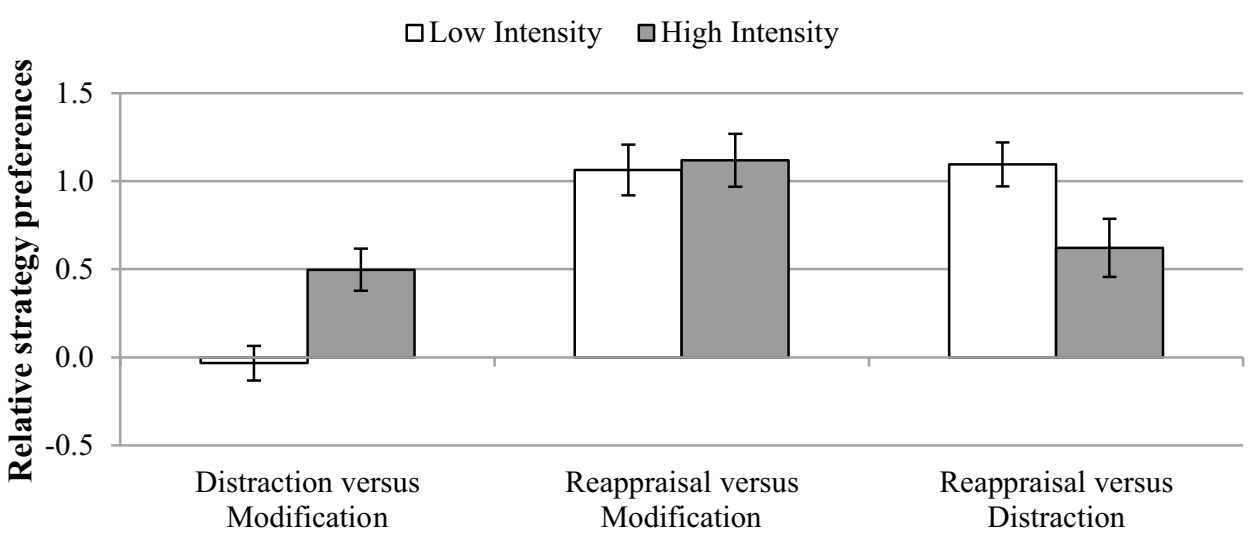

Strategy comparison pair

\section{Negative emotion downregulation effect}

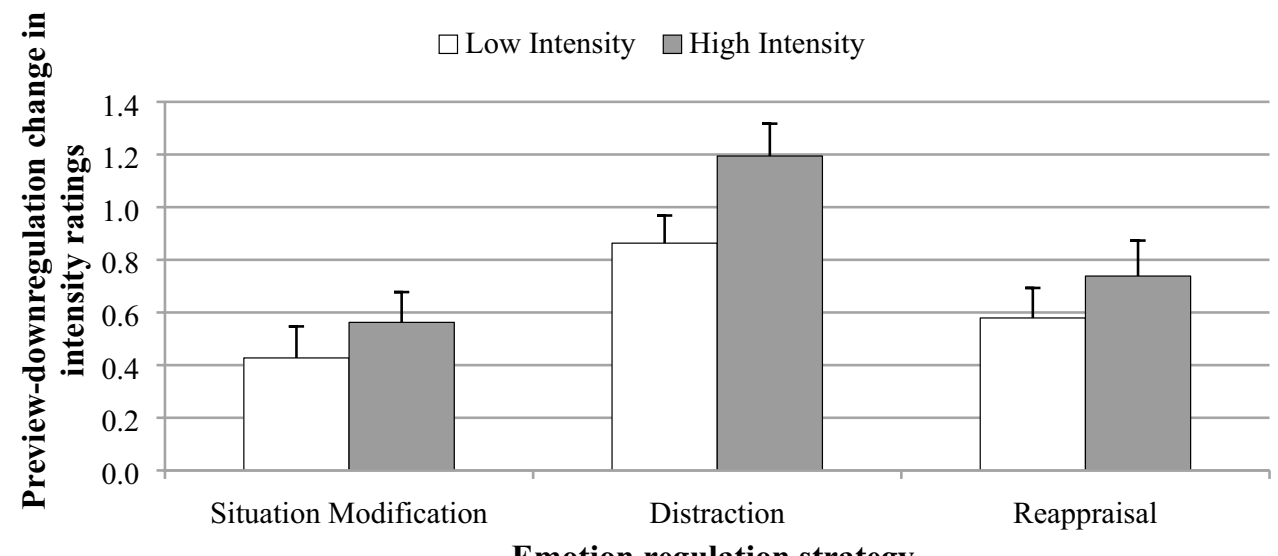

Emotion regulation strategy 
(Aitchison 2003). ${ }^{1}$ To avoid undefined outcomes caused by division by zero (in case participants never selected a certain strategy ${ }^{2}$ ) in our calculation of these relative preferences, the observed frequencies were first transformed following Agresti and Coull's (1998) formula: (observed frequency +2$) /($ number of observations +4$)$. Next, we calculated three relative strategy preferences by (1) dividing the choice frequencies for distraction by the choice frequencies for situation modification, (2) by dividing the choice frequencies for reappraisal by the choice frequencies for situation modification, and (3) by dividing the choice frequencies for reappraisal by the choice frequencies for distraction. These scores thus indicated a preference for one strategy over another strategy, ignoring the choice frequency of the third strategy. Finally, we calculated the natural logarithm of these relative frequencies. Positive values reflect a preference for the first-named strategy in each comparison and negative values reflect a preference for the second-named strategy in each comparison.

To address the effectiveness of the strategies, negative emotion intensity ratings were calculated by averaging the four different negative emotion scales (fear, disgust, sadness, and anger: Cronbach's alpha for preview ratings across all trials $=.86$; Cronbach's alpha for downregulation ratings across all trials $=.89$ ), separately for the preview and downregulation phase, as well as for low versus high-intensity trials and each strategy choice. Reflecting our distinction between high and low-intensity picture pairs, participants rated the high-intensity preview displays $(M=5.20$, $S D=1.61)$ as more intensely negative than the low-intensity preview displays $(M=3.21, S D=1.33), F(1,37)=194.04$, $p<.001$.

For the flanker task, the data of one participant were set to missing because he or she made too many errors (participant's score $=53.33 \%$ correct, group mean $=94.82$, $S D=8.62)$. Next, we removed errors $(4.0 \%)$, trials with reaction times (RTs) deviating more than three $S D$ s from the group mean $(M=433.88, S D=113.55$, cut-off $=774.53$, $1.5 \%$ removed), and trials with RTs deviating more than three $S D$ s from each individual's mean (1.4\% removed). From the remaining data, we calculated congruency effects

\footnotetext{
${ }^{1}$ In compositional data, data points reflect a proportion or percentage of some whole, meaning that their sum is constrained to be a certain constant (e.g., in the case of percentages, the sum of all observed percentages typically equals $100 \%$ ). Compositional data cannot be analysed correctly with standard statistical approaches, unless they are transformed into log-ratios prior to analysis.

2 Out of the 38 participants of Experiment 1, the numbers of participants who never selected a particular strategy on either stimulus intensity were as follows: Distraction low intensity: $\mathrm{N}=4$; Distraction high intensity: $\mathrm{N}=3$; Modification low intensity: $\mathrm{N}=3$; Modification high intensity: $\mathrm{N}=8$; Reappraisal low intensity: $\mathrm{N}=0$; Reappraisal high intensity: $\mathrm{N}=0$.
}

by subtracting each participant's average RT on congruent trials from their average RT on incongruent trials.

\section{Strategy choices and effects of stimulus intensity on strategy choices}

The raw choice frequencies in Fig. 3a illustrate that participants distributed their strategy choices in both high and low-intensity trials over the three options and-achieving our main goal of the study - that participants did choose to use our situation modification option to regulate their emotions. Confirming the idea that stimulus intensity influences strategy choices, paired samples $t$ test showed that distraction was chosen more often for high compared to low-intensity trials, $t(37)=3.75, p=.001, \mathrm{CI}_{\text {difference }}=[5.59$, $18.70], d=0.61$, while reappraisal was chosen more often for low compared to high-intensity trials, $t(37)=2.09, p=.043$, $\mathrm{CI}_{\text {difference }}=[0.25,14.94], d=0.34$. Contrary to our hypothesis, choices for situation modification were not significantly affected by stimulus intensity, $t(37)=1.75, p=.088$, $\mathrm{CI}_{\text {difference }}=[-0.72,9.83], d=0.28$.

\section{Effects of stimulus intensity on relative strategy preferences}

Our next question concerned the relative preferences of one strategy over another, and whether these preferences also depended on stimulus intensity. To answer this question, two of the three relative strategy preferences (the third relative preference being redundant for the multivariate effect) were analysed together in a single repeated measures MANOVA, with Stimulus Intensity (low vs. high) as a within-subjects factor. The multivariate effect of Stimulus Intensity was significant, $F(2,36)=8.13$, $p=.001, f=0.67$, indicating that, overall, preferences for one strategy over another were affected by stimulus intensity. Follow-up univariate analyses (Fig. 3b) on each of the three relative preferences separately revealed significant effects of Stimulus Intensity in the comparison between situation modification and distraction, $F(1,37)=14.64$, $p<.001, \mathrm{CI}_{\text {difference }}=[0.25,0.81], f=0.63$, and in the comparison between distraction and reappraisal, $F(1,37)=9.84$, $p=.003, \mathrm{CI}_{\text {difference }}=[0.17,0.78], f=0.52$, but not in the comparison between situation modification and reappraisal, $F(1,37)=0.15, p=.704, \mathrm{CI}_{\text {difference }}=[-0.35,0.24]$. These effects indicate that distraction was more popular for high rather than low-intensity stimuli, at the cost of both situation modification and reappraisal. Preferences for situation modification at the cost of reappraisal were not affected by stimulus intensity (i.e., reappraisal was preferred over situation modification to a similar extent for both low and high intensity stimuli). Finally, comparing the relative frequencies with zero (values not differing from zero would indicate no preference for either strategy), one-sample $t$-tests 
showed that reappraisal was preferred over distraction for both high, $t(37)=3.77, p<.001, \mathrm{CI}_{\text {difference }}=[0.29,0.96]$, $d=0.61$, and low-intensity stimuli, $t(37)=8.79, p<.001$, $\mathrm{CI}_{\text {difference }}=[0.84,1.35], d=1.43$, reappraisal was also preferred over situation modification for both high, $t(37)=7.43$, $p<.001, \mathrm{CI}_{\text {difference }}=[0.81,1.42], d=1.21$, and low-intensity stimuli, $t(37)=7.38, p<.001, \mathrm{CI}_{\text {difference }}=[0.77,1.36]$, $d=1.20$, and distraction was preferred over situation modification for high-intensity stimuli, $t(37)=4.18, p<.001$, $\mathrm{CI}_{\text {difference }}=[0.26,0.74], d=0.68$, but not low-intensity stimuli, $t(37)=0.34, p=.735, \mathrm{CI}_{\text {difference }}=[-0.23,0.17]$, $d=0.06$.

\section{Emotion regulation effectiveness}

To address the effectiveness of each of the strategies, negative emotion intensity ratings were analysed in a repeated measures ANOVA, with Time (preview vs. downregulation), Stimulus Intensity, and Strategy Choice (situation modification vs. distraction vs. reappraisal) as within-subjects factors. While interactions involving Time and Strategy Choice would imply that certain strategies were more effective than others, this analysis should be interpreted with caution, because the downregulation ratings were likely influenced by the choice of strategy (i.e., participants more likely chose the strategy that they anticipated would work best in the given circumstances). The ANOVA yielded significant main effects of Time and Stimulus Intensity, $F(1,25)=67.26, p<.001, f=1.64$, and $F(1,25)=94.05, p<.001, f=1.94$, respectively, merely reflecting the instructed downregulation effect and our manipulation of low versus high-intensity stimuli. These main effects were qualified by the interactions between Time and Stimulus Intensity, $F(1,25)=5.43, p=.028$, $f=0.47$, Time and Strategy Choice, $F(2,24)=14.43$, $p<.001, f=1.10$, and Stimulus Intensity and Strategy Choice, $F(2,24)=5.96, p=.008, f=0.70$. No other effects were significant, all $F \mathrm{~s}<1.90$, all $p \mathrm{~s}>.172$, all $f_{s}<0.40$. To follow-up on the two interactions involving the factor Time, we calculated change scores by subtracting the downregulation ratings from the preview ratings (Fig. 3c), separately for each picture intensity and each strategy choice. Bonferroni-corrected contrast comparisons showed stronger downregulation in response to high compared to low-intensity pictures, $t(25)=3.08$, $p=.003, \mathrm{CI}_{\text {difference }}=[0.02,0.39], d=0.61$. In addition, distraction led to more downregulation than both situation modification, $t(25)=6.56, p<.001, \mathrm{CI}_{\text {difference }}=[0.28$, $0.79], d=1.29$, and reappraisal, $t(25)=4.33, p<.001$, $\mathrm{CI}_{\text {difference }}=[0.13,0.61], d=0.85$, with no difference between the latter two strategies, $t(25)=2.26, p=.085$, $\mathrm{CI}_{\text {difference }}=[-0.05,0.38], d=0.44$.
Exploratory relations between emotion regulation preferences and individual characteristics

To explore the relations between strategy preferences and levels of depression, self-reported strategy use, and cognitive control, we conducted three separate MANOVAs on two of the three relative strategy preferences, irrespective of stimulus intensity (see also Supplemental Materials, section Results Experiment 1). The MANOVA with the BDI as a continuous predictor yielded no significant multivariate effect, $F(2,35)=0.86, p=.433$, indicating that strategy preferences were not related to self-reported levels of depression. In a similar vein, the MANOVA with Positive refocusing, Refocus on planning, Positive reappraisal, and Putting into perspective as continuous predictors yielded no significant multivariate effects, all $F \mathrm{~s}<2.39$, all $p \mathrm{~s}>.108$, suggesting that strategy preferences in the lab are not necessarily reflected by self-reported trait strategy use. Finally, the MANOVA with the flanker congruency effect as a continuous predictor yielded no significant result, $F(2,35)=0.69$, $p=.511$, indicating that our measure of cognitive control was not associated with strategy preferences.

\section{Discussion}

Confirming our expectations, participants chose to modify their situation in order to regulate their emotions, although they did not do so more often for high compared to lowintensity stimuli. Irrespective of stimulus intensity, situation modification was less effective than distraction but did not differ from reappraisal. In line with the findings of Sheppes et al. (2011, 2014), distraction became more popular with increased stimulus intensity, although even for high intensity trials distraction was not preferred over reappraisal. This overall strong preference for reappraisal could be due to our cues reducing the complexity of generating reappraisals (Sheppes et al. 2014, Experiment 2). Presenting cues for distraction and reappraisal could also be considered a limitation, as they arguably modified the stimulus display and thus the situation. Therefore, in Experiment 2, we omitted the reappraisal and distraction cues, resulting in more straightforward and unbiased measures of all three emotion regulation strategies.

\section{Experiment 2}

\section{Method}

\section{Participants}

Thirty-eight students ( 26 women, $M_{\text {age }}=23.16, S D=4.51$, range $=18-42$ ) of the University of Amsterdam participated 
in Experiment 2 in exchange for course credits or $€ 10$. None of the participants had also participated in Experiment 1, and the sample size was based on the same power analysis as in Experiment 1.

\section{Questionnaires, materials, tasks, and procedure}

The experimental design of Experiment 2 was identical to the design of Experiment 1, the only exception being the absence of cues when participants chose distraction or reappraisal in the emotion regulation choice task. When participants selected distraction or reappraisal, the same picture pair as in the preview phase was presented on the screen for $10,000 \mathrm{~ms}$, and participants were instructed to implement the strategy that they had chosen. In order to make sure that participants understood both the instructions (see Supplemental Materials, section Methods Experiment 2) and each of the strategies, an experimenter stayed in the room during the entire emotion regulation practice phase. After each picture pair in the practice phase, participants were asked to verbally explain to the experimenter what they had done to downregulate their negative emotions (i.e., point at the neutral part of the stimulus display that they had focused their attention on or verbalize the reappraisal that they had made). When necessary, the experimenter corrected the use of strategies by explaining any errors and giving one or more extra examples, and restarted the practice block to give participants the chance to practice the strategies correctly. After this, the test phase of the emotion regulation choice task started.

Cronbach's alphas of the questionnaires and subscales in Experiment 2 were as follows: CERQ: Positive refocusing: .74, Refocus on planning: .77, Positive reappraisal: .82, Putting into perspective: .65; BDI-II: .89 (for details of exploratory questionnaires that were included in the study, see Supplemental Materials, section Methods Experiment 2).

\section{Results}

\section{Data reduction and scoring}

The scoring of the emotion regulation data was identical to the procedure described for Experiment 1 (Cronbach's alpha for preview emotion ratings across all trials $=.87$; Cronbach's alpha for downregulation emotion ratings across all trials $=.92) .{ }^{3}$ Participants rated the

\footnotetext{
${ }^{3}$ Out of the 38 participants of Experiment 2, the numbers of participants who never selected a particular strategy on either stimulus intensity were as follows: Distraction low intensity: $\mathrm{N}=1$; Distraction high intensity: $\mathrm{N}=1$; Modification low intensity: $\mathrm{N}=3$; Modification high intensity: $\mathrm{N}=3$; Reappraisal low intensity: $\mathrm{N}=0$; Reappraisal high intensity: $\mathrm{N}=0$.
}

high-intensity preview displays $(M=4.55, S D=1.76)$ again as more intensely negative than the low-intensity preview displays $(M=2.72, S D=1.14), F(1,37)=146.60$, $p<.001$. For the flanker task, we also used the same outlier analysis: We first removed errors (7.7\%) and the data of one participant were set to missing because he or she was on average very slow to respond (group $M=480.11$, $S D=196.91$, participant's $M=1538.34$ ). Next, we removed trials with RTs deviating more than three $S D$ s from the group mean $(M=451.55, S D=178.58$, cutoff $=987.29,1.5 \%$ removed , and trials with RTs deviating more than three $S D$ s from each individual's mean $(1.4 \%$ removed). We calculated congruency effects by subtracting each participant's average RT on congruent trials from their average RT on incongruent trials.

\section{Strategy choices and effects of stimulus intensity on strategy choices}

The raw strategy choice frequencies were distributed over the three options (Fig. 4a), again achieving our first goal of creating a paradigm in which participants modified their situation in order to regulate their emotions. In line with our original hypothesis, paired samples $t$-tests showed that both situation modification and distraction were chosen more often for high compared to low-intensity trials, $t(37)=2.10$, $p=.043, \mathrm{CI}_{\text {difference }}=[0.27,15.31], d=0.34$, and $t(37)=2.17$, $p=.036, \mathrm{CI}_{\text {difference }}=[0.60,17.42], d=0.35$, respectively. In contrast, reappraisal was chosen more often for low compared to high-intensity trials, $t(37)=5.45, p<.001$, $\mathrm{CI}_{\text {difference }}=[10.56,23.05], d=0.88$.

\section{Effects of stimulus intensity on relative strategy preferences}

As in Experiment 1, our next question concerned the effects of stimulus intensity on the relative preferences of one strategy over another. We ran a repeated measures MANOVA on two of the three relative strategy preferences, with Stimulus Intensity as a within-subjects factor. The multivariate effect of Stimulus Intensity was again significant, $F(2,36)=15.17$, $p<.001, f=0.92$, confirming that preferences for one strategy over another were affected by stimulus intensity. Followup univariate analyses (Fig. 4b) on each of the three relative preferences separately revealed significant effects of Stimulus Intensity in the situation modification versus reappraisal comparison, $F(1,37)=18.73, p<.001, \mathrm{CI}_{\text {difference }}=[0.35$, $0.97], f=0.71$, and in the distraction versus reappraisal comparison, $F(1,37)=18.33, p<.001, \mathrm{CI}_{\text {difference }}=[0.34$, $0.95], f=0.70$, but not in the comparison between situation modification and distraction, $F(1,37)=0.00, p=.944$, $\mathrm{CI}_{\text {difference }}=[-0.38,0.40], f=0.00$. Thus, when participants had no reappraisal or distraction cues to downregulate negative emotions, participants became more likely to choose 
Fig. 4 Summary of emotion regulation results of Experiment 2, separating high and low-intensity stimuli. a Shows raw strategy choice percentages, separately for each strategy. b Shows relative strategy preferences for each of the three strategy comparisons. Values represent the log-transformed preference of the first-named strategy in each pair relative to the second-named strategy in each pair. Positive values reflect a preference for the first-named strategy in each comparison and negative values reflect a preference for the second-named strategy in each comparison. Inverting these values-i.e., calculating $\operatorname{EXP}(\mathrm{x})$ - yields the untransformed preferences (e.g., the value of around 0.5 in the comparison between reappraisal and situation modification on low intensity trials indicates that reappraisal was chosen about $\operatorname{EXP}(0.5)=1.6$ times more often than situation modification). $\mathbf{c}$ Shows the self-reported emotion downregulation effect (i.e., preview ratings minus downregulation ratings), separately for each strategy. Large positive values thus indicate more downregulation. Error bars reflect standard errors of the mean in all panels

\section{A Strategy choice frequencies}

$\square$ Low Intensity $\square$ High Intensity

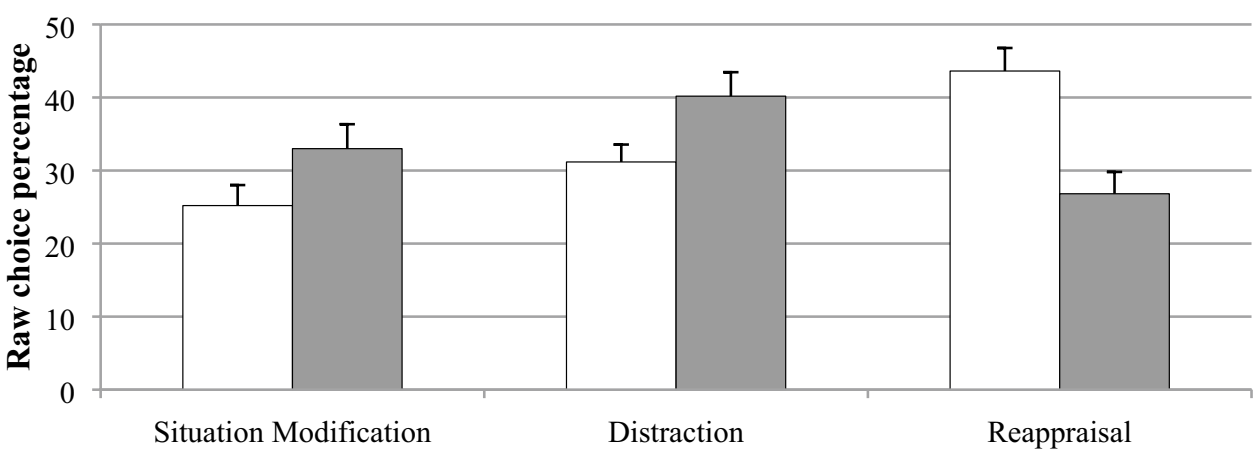

Emotion regulation strategy choice

\section{B Relative strategy preferences}

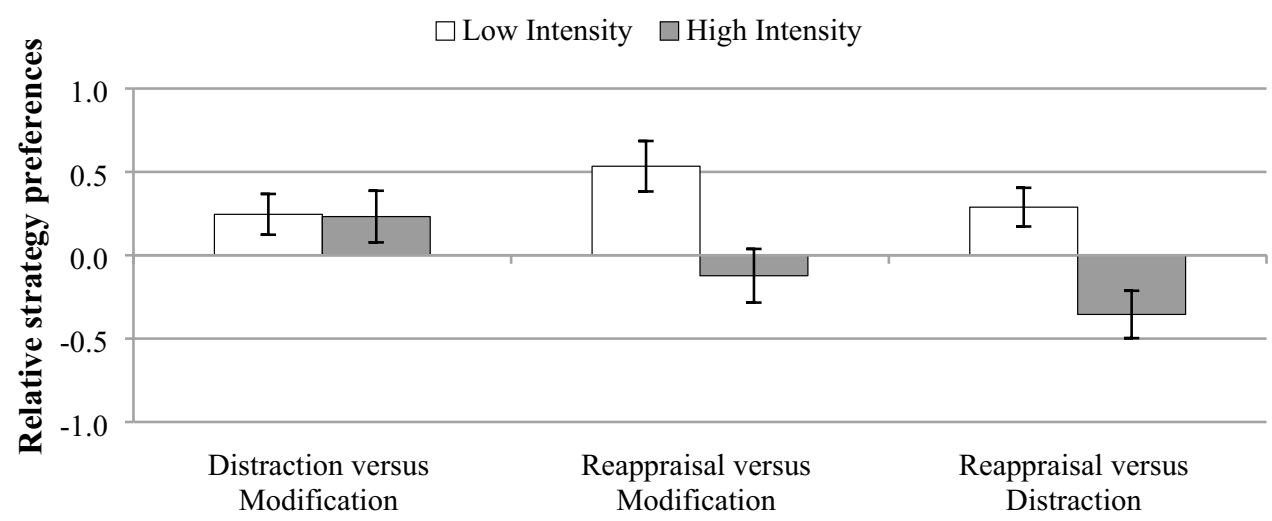

Strategy comparison pair

\section{Negative emotion downregulation effect}

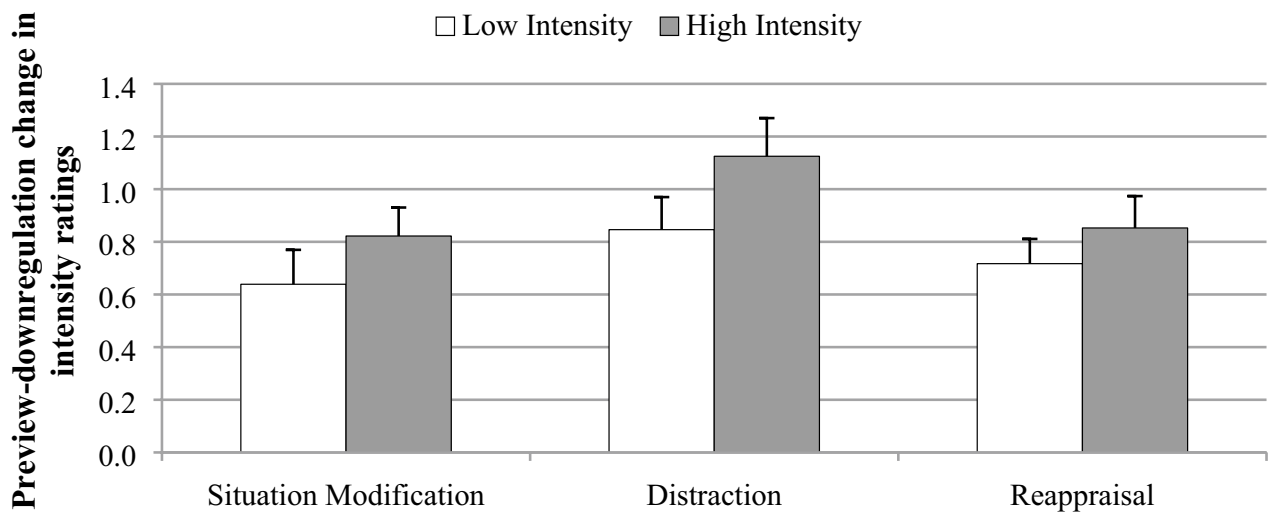

Emotion regulation strategy distraction or situation modification with increased stimulus intensity, at the cost of reappraisal. Preferences for situation modification versus distraction were not affected by stimulus intensity (i.e., increased stimulus intensity did not make people more likely to choose either strategy at the cost of the other).

One-sample $t$-tests comparing the relative frequencies with zero showed that for low-intensity stimuli, reappraisal 
was preferred over both distraction, $t(37)=2.49, p=.018$, $\mathrm{CI}_{\text {difference }}=[0.05,0.52], d=0.40$, and situation modification, $t(37)=3.53, p=.001, \mathrm{CI}_{\text {difference }}=[0.23,0.84], d=0.57$. For high-intensity stimuli, distraction was preferred over reappraisal, $t(37)=2.50, p=.017, \mathrm{CI}_{\text {difference }}=[0.07,0.64]$, $d=0.41$. There was no preference for distraction relative to situation modification for either low, $t(37)=2.01, p=.052$, $\mathrm{CI}_{\text {difference }}=[0.00,0.49], d=0.33$, or high-intensity stimuli, $t(37)=1.50, p=.143, \mathrm{CI}_{\text {difference }}=[-0.08,0.55], d=0.24$, and no preference for situation modification over reappraisal for high-intensity stimuli, $t(37)=0.76, p=.450$, $\mathrm{CI}_{\text {difference }}=[-0.20,0.45], d=0.12$.

\section{Emotion regulation effectiveness}

Addressing the effectiveness of each of the strategies, we conducted a repeated measures ANOVA on the average negative emotion intensity ratings with Time, Stimulus Intensity, and Strategy Choice as within-subjects factors. We again found significant main effects of Time, $F(1$, $33)=107.25, p<.001, f=1.80$, and Stimulus Intensity, $F(1,33)=88.45, p<.001, f=1.64$. We also found a main effect of Strategy Choice, $F(2,32)=3.44, p=.044, f=0.46$. These main effects were qualified by significant interactions between Time and Stimulus Intensity, $F(1,33)=4.97$, $p=.033, f=0.39$, and between Stimulus Intensity and Strategy Choice, $F(2,32)=4.46, p=.020, f=0.53$. As in Experiment 1 , the Time by Stimulus Intensity interaction reflected more downregulation for high compared to low-intensity pictures, $t(33)=2.66, p=.009, \mathrm{CI}_{\text {difference }}=[0.02,0.38], d=0.46$ (Bonferroni corrected). Following-up on the Stimulus Intensity by Strategy Choice interaction, paired samples $t$-tests showed that for low-intensity pictures (averaged across Time), people rated displays for which they chose distraction $(M=2.55, S D=1.07)$ as more intense than displays for which they chose to use reappraisal $(M=2.28, S D=1.04)$, $t(36)=3.08, p=.004$. Comparisons between distraction and situation modification $(M=2.51, S D=1.32)$ and between reappraisal and situation modification were not significant, both $t \mathrm{~s}<1.55$, both $p \mathrm{~s}>.13$. For high intensity pictures, people rated stimulus displays for which they chose situation modification $(M=4.23, S D=1.87)$ as more intense than displays for which they chose either distraction $(M=3.88$, $S D=1.71), t(34)=2.81, p=.008$, or reappraisal $(M=3.76$, $S D=1.74), t(34)=3.28, p=.002$. There was no difference between displays for which they chose distraction versus displays for which they chose reappraisal, $t(36)<1, p=.58$. Crucially however, neither the interaction between Time and Strategy Choice, $F(2,32)=2.63, p=.087, f=0.41$, nor the three-way interaction, $F(2,32)=0.37, p=.691, f=0.15$, were significant, indicating that there were no significant differences between the strategies in their downregulation effectiveness (Fig. 4c).
Exploratory relations between emotion regulation preferences and individual characteristics

As in Experiment 1, we explored whether total emotion regulation strategy preferences were associated with levels of depression, self-reported strategy use, and cognitive control in three separate MANOVAs (see also Supplemental Materials, section Results Experiment 2). None of these MANOVAs yielded significant multivariate effects: BDIII: $F(2,35)=1.20, p=.314$; CERQ subscales: all $F \mathrm{~s}<1.58$, all $p \mathrm{~s}>.221$; flanker congruency effect: $F(2,34)=1.92$, $p=.162$. As such, we found no support for the ideas that strategy preferences relate to depression, self-reported trait strategy use, or cognitive control.

\section{Discussion}

Omitting the cues for distraction and reappraisal, people again chose situation modification to successfully downregulate negative affect, and in line with our hypothesis, they did so more often for high than for low-intensity displays. Further, reappraisal was preferred over both distraction and situation modification for low-intensity displays, while in line with the original findings of Sheppes et al. (2011), distraction was preferred over reappraisal for high-intensity displays. Relative to Experiment 1, omitting the cues for reappraisal made reappraisal less popular, probably due to the increased complexity of generating reappraisals oneself (see also Sheppes et al. 2014). Finally, we found no differences between strategies in their effectiveness.

\section{General discussion}

Based on Gross' $(1998,2015)$ emotion regulation model, we developed a paradigm that allowed participants to modify a part of their emotional environment to downregulate their negative affect, without avoiding the emotional situation entirely. In two experiments, we found that participants did use this strategy when given the choice between situation modification, distraction, and reappraisal. Although the emotion intensity ratings were likely influenced by participants' expected utility of the strategies that they chose, we found no significant differences in effectiveness between the three strategies in Experiment 2. Our paradigm thus presents a first step in the systematic lab-based study of emotion regulation through situation modification.

Our results also provide further support for the idea that emotion regulation strategy choices are affected by the emotional intensity of the stimuli on display. In both experiments, we replicated Sheppes et al.'s $(2011,2014)$ findings of increased choices for distraction relative to reappraisal for high compared to low-intensity negative stimuli. The 
results of Experiment 2 extend these findings, revealing that situation modification was also chosen more often for high compared to low-intensity stimuli. Our results are therefore in line with the general idea that disengagement-focused strategies (such as avoidance, situation modification, and distraction) are preferred for high-intensity stimuli (see also Vujovic et al. 2014), while engagement-focused strategies (such as reappraisal) are preferred for low-intensity stimuli.

Although it has been argued that strategy preferences reflect the effectiveness of strategies (Gross 2015; but see Urry and Gross 2010), our strategy preference effects were not mirrored in the downregulation ratings, and we found only limited differences in strategy effectiveness. As studies using no-choice paradigms have indicated that distraction is more effective than reappraisal to downregulate intense negative emotions (e.g., Shafir et al. 2015), our limited differences in effectiveness were likely due to participants choosing strategies that they expected to be effective. Moreover, the relatively long picture presentation durations in the preview phase may have enabled participants to spontaneously start regulating emotions before being asked to do so. In addition, relying solely on self-reports, the effectiveness results could have been influenced by demand effects. A design with instructed strategy use, also including physiological or behavioural measures of emotion intensity, would be crucial to unambiguously compare the effectiveness of situation modification with other strategies. Adding a nodownregulation control group could further differentiate between the effects of intentional downregulation and mere exposure or habituation.

Contrary to our exploratory hypotheses, none of the strategy preferences were related to levels of depression (Haines et al. 2016; Troy et al. 2013) or cognitive control (Urry and Gross 2010). This lack of associations could be explained by our finding that none of the strategy preferences in the lab were related to self-reported trait strategy use as measured by the CERQ. To our knowledge, only one previous study assessed strategy preferences using both self-report and a behavioural choice paradigm: In line with our findings, Sauer et al. (2016) found that differences in self-reported reappraisal and distraction use were not reflected in behavioural choices in the lab. This lack of correlations between self-reported strategy use and lab-based strategy choices could in part be explained by the fact that strategy choice paradigms include only a limited amount of possible strategies. If participants do not use any of these strategies in their daily life, their forced choice of one of these strategies in the lab may blur the pattern of correlations. Alternatively, there may be a genuine dissociation between lab-based and reallife emotion regulation strategy preferences. Such a dissociation could imply that the ecological validity of lab-based strategy choice paradigms is poor, but it might also indicate that people lack insight in which strategies they use and how often they do so in real life, biasing their response patterns in questionnaires. For instance, Ford et al. (2017) found only modest correlations of around .20 between reappraisal use in a questionnaire and reappraisal frequency and success in daily diary assessments, suggesting that questionnaires may yield relatively imprecise estimates of reappraisal use. With respect to our current findings, the lack of significant relation between lab-based and self-reported strategy choices may also reflect a power issue. As our power analysis focussed on detecting medium to large effects of stimulus intensity on strategy choice, larger studies should further address (interactions between) individual and situational determinants of strategy choice.

Our operationalization of situation modification paves the way for future research. Unlike most cases of situation modification in real life, our present situation modification manipulation did not require any real effort and did not come at a potential cost. Future studies could address whether the frequency and effectiveness of situation modification are affected by the cost of changing the situation, for instance by requiring participants to actively invest time, resources, or energy in situation modification. Further manipulations could include varying the number of aspects in a situation that can be changed, allowing participants to exert control over which aspects of the situation they want to change, or including trials in which attempted situation modification leads to a positive aspect rather than merely reducing the intensity of a negative aspect, trials in which situation modification is not successful, or even trials in which attempting to modify the situation leads to a worse outcome. Given the negative relation between psychopathology and self-reported use of situation modification (Aldao et al. 2010), studies with clinical samples or participants who are exposed to stressors could address the relation between psychopathology and situation modification choices. Studies with more trials may add more possible strategies for participants to choose from, or may even be open-ended, allowing participants to regulate their emotions in any way they see fit. Because each choice percentage in our study depended on the popularity of the other strategies (illustrated for instance by the overall popularity of reappraisal in Experiment 1) and was also confined by the strategies on offer, such open-ended paradigms would allow for a more unambiguous interpretation of strategy choice percentages.

As for our stimulus selection, we differentiated between high and low-intensity pictures based on the normative ratings of the IAPS (Lang et al. 2008). Although the preview ratings in our data were in line with this differentiation, negative IAPS pictures tend to confound valence and arousal, with highly negative pictures typically also being more arousing, and moderately negative pictures typically also being only moderately arousing. As such, we cannot attribute the stimulus intensity effects to arousal alone: 
High-intensity pictures should be seen as a combination of high arousal and strong negative valence, while low-intensity pictures should be seen as a combination of moderate arousal and moderate negative valence.

We always presented two pictures on the screen to isolate situation modification from situation selection or avoidance. However, as situation modification choices affected only one picture, situation modification in our study could be considered as only partially effective, possibly decreasing the popularity or effectiveness of the strategy. Related to this issue, our operationalization of situation modification, using a fading-effect on part of the stimulus display, may be somewhat artificial and may have also influenced participants' attention. An alternative manner of changing emotional stimulus displays while not avoiding them altogether could be to zoom in or to zoom out on the stimulus display, thus mimicking a decrease or increase in the physical distance between oneself and the display (e.g., see Davis et al. 2011).

A fundamental limitation concerns the use of multiple strategies. Although previous studies have shown that participants do generally use the strategies of their choice (Sheppes et al. 2011), we did not check whether they did so in our study. It is thus possible that participants engaged in different or even multiple emotion regulation strategies, either simultaneously or sequentially (Gross 2015). Such use of follow-up strategies may have especially been the case in the downregulation period following the modification of the stimulus display. The regulatory effects of situation modification may thus reflect the sum or combination of modifying the situation and any follow-up strategies that were used, possibly also distraction and/or reappraisal. Failing to fully isolate unique emotion regulation strategies is likely a recurring problem in many emotion regulation studies. When attempting to reappraise a picture of a traffic accident by thinking about how paramedics will save everyone involved, it is not unlikely that people will at the same time redirect their attention to for instance an ambulance in the background of the scene (e.g., see Van Reekum et al. 2007; but see also Urry 2010). While combined strategy use may thus have affected emotion regulation, especially when participants opted for situation modification, it is also important to note that in Experiment 2, the stimulus display only changed when choosing situation modification. As such, the effects of physically altering the emotion-evoking display on emotion regulation were still unique to those trials where people opted for situation modification. In future studies, asking participants to describe their emotion regulation strategy out loud during the downregulation phase could confirm whether participants do indeed stick to the strategy of their choice. In addition, a shorter downregulation phase could diminish the chance of participants using multiple strategies.

Despite these limitations, our study adds to the literature in several ways. Research on situation modification is scarce and has typically confounded situation modification with avoidance. With recent studies showing that other emotion regulation strategies may only be adaptive in uncontrollable situations, a solid understanding of situation modification as an emotion regulation strategy is crucial. We developed and tested a novel operationalization of situation modification that does not confound situation modification with avoidance or situation selection. Participants chose to modify stimulus displays to successfully downregulate negative emotions. Furthermore, we confirmed that emotion regulation strategy preferences are influenced by the intensity of emotional situations, with disengagement-focused strategies (situation modification and distraction) being preferred for high-intensity stimuli and engagement-focused strategies (reappraisal) being preferred for low-intensity stimuli.

Acknowledgements Bram Van Bockstaele is a postdoctoral researcher of Research Priority Area Yield of the University of Amsterdam, and is partially supported by Sara van Dam z.l. Foundation, Royal Netherlands Academy of Arts and Sciences. Helle Larsen is partly funded by Research Priority Area Yield of the University of Amsterdam. We thank Robert Zwitser, Raoul Grasman, and Sophia van Ghesel-Grothe for statistical consulting and checks, and Gal Sheppes for sharing stimulus materials.

Data availability Neither of the experiments reported in this article was formally preregistered. The original scripts (except for the copyrighted IAPS pictures), raw data, transformed data, and results that were used in Experiment 1 and Experiment 2 are accessible in the following Open Science Framework data deposit: https://doi.org/10.17605 /OSF.IO/3NBEC.

\section{Compliance with ethical standards}

Conflict of interest The authors declare that they have no conflict of interest.

Ethical approval All procedures performed in studies involving human participants were in accordance with the ethical standards of the institutional and/or national research committee and with the 1964 Helsinki declaration and its later amendments or comparable ethical standards.

Open Access This article is distributed under the terms of the Creative Commons Attribution 4.0 International License (http://creativeco mmons.org/licenses/by/4.0/), which permits unrestricted use, distribution, and reproduction in any medium, provided you give appropriate credit to the original author(s) and the source, provide a link to the Creative Commons license, and indicate if changes were made.

\section{References}

Agresti, A., \& Coull, B. A. (1998). Approximate is better than "exact" for interval estimation of binomial proportions. The American Statistician, 52, 119-126. https://doi.org/10.2307/2685469.

Aitchison, J. (2003). The statistical analysis of compositional data. London: Blackburn Press.

Aldao, A., Nolen-Hoeksema, S., \& Schweizer, S. (2010). Emotionregulation strategies across psychopathology: A meta-analytic 
review. Clinical Psychology Review, 30, 217-237. https://doi. org/10.1016/j.cpr.2009.11.004.

Aldao, A., Sheppes, G., \& Gross, J. J. (2015). Emotion regulation flexibility. Cognitive Therapy and Research, 39, 263-278. https://doi. org/10.1007/s 10608-014-9662-4.

Beck, A. T., Steer, R. A., \& Brown, G. K. (1996). Manual for the Beck depression inventory-II. San Antonio, TX: Psychological Corporation.

Bonanno, G. A., \& Burton, C. L. (2013). Regulatory flexibility: An individual differences perspective on coping and emotion regulation. Perspectives on Psychological Science, 8, 591-612. https:// doi.org/10.1177/1745691613504116.

Cohen, J. (1992). A power primer. Psychological Bulletin, 112, 155159. https://doi.org/10.1037/0033-2909.112.1.155.

Davis, J. I., Gross, J. J., \& Ochsner, K. N. (2011). Psychological distance and emotional experience: What you see is what you get. Emotion, 11, 438-444. https://doi.org/10.1037/a0021783.

Eriksen, B. A., \& Eriksen, C. W. (1974). Effects of noise letters upon the identification of a target letter in a nonsearch task. Attention, Perception, \& Psychophysics, 16, 143-149. https://doi. org/10.3758/BF03203267.

Faul, F., Erdfelder, E., Lang, A. G., \& Buchner, A. (2007). G*Power 3: A flexible statistical power analysis program for the social, behavioral, and biomedical sciences. Behavior Research Methods, 39, 175-191. https://doi.org/10.3758/BF03193146.

Ford, B. O., Karnilowicz, H. R., \& Mauss, I. B. (2017). Understanding reappraisal as a multicomponent process: The psychological health benefits of attempting to use reappraisal depend on reappraisal success. Emotion, 17, 905-911. https://doi.org/10.1037/ emo0000310.

Garnefski, N., Kraaij, V., \& Spinhoven, P. (2001). Negative life events, cognitive emotion regulation, and emotional problems. Personality and Individual Differences, 30, 1311-1327. https://doi. org/10.1016/S0191-8869(00)00113-6.

Gross, J. J. (1998). The emerging field of emotion regulation: An integrative review. Review of General Psychology, 2, 271-299. https ://doi.org/10.1037/1089-2680.2.3.271.

Gross, J. J. (2015). Emotion regulation: Current status and future prospects. Psychological Inquiry, 26, 1-26. https://doi. org/10.1080/1047840X.2014.940781.

Haines, S. J., Gleeson, J., Kuppens, P., Hollenstein, T., Ciarrochi, J., Labuschagne, I., ... Koval, P. (2016). The wisdom to know the difference: Strategy-situation fit in emotion regulation in daily life is associated with well-being. Psychological Science, 27, 1651-1659. https://doi.org/10.1177/0956797616669086.

Lang, P. J., Bradley, M. M., \& Cuthbert, B. N. (2008). International affective picture system (IAPS): Affective ratings of pictures and instruction manual. Technical Report A-8. University of Florida, Gainesville, FL.

Livingstone, K. M., \& Isaacowitz, D. M. (2015). Situation selection and modification for emotion regulation in younger and older adults. Social Psychological and Personality Science, 6, 904-910. https://doi.org/10.1177/1948550615593148.

Opitz, P. C., Rauch, L. C., Terry, D. P., \& Urry, H. L. (2012). Prefrontal mediation of age differences in cognitive reappraisal.
Neurobiology of Aging, 33, 645-655. https://doi.org/10.1016/j. neurobiolaging.2010.06.004.

Sauer, C., Sheppes, G., Lackner, H. K., Arens, E. A., Tarrasch, R., \& Barnow, S. (2016). Emotion regulation choice in female patients with borderline personality disorder: Findings from self-reports and experimental measures. Psychiatry Research, 242, 375-384. https://doi.org/10.1016/j.psychres.2016.04.113.

Shafir, R., Schwartz, N., Blechert, J., \& Sheppes, G. (2015). Emotional intensity influences pre-implementation and implementation of distraction and reappraisal. SCAN, 10, 1329-1337. https://doi. org/10.1093/scan/nsv022.

Sheppes, G., Scheibe, S., Suri, G., \& Gross, J. J. (2011). Emotionregulation choice. Psychological Science, 22, 1391-1396. https ://doi.org/10.1177/0956797611418350.

Sheppes, G., Scheibe, S., Suri, G., Radu, P., Blechert, J., \& Gross, J. J. (2014). Emotion regulation choice: A conceptual framework and supporting evidence. Journal of Experimental Psychology: General, 143, 163-181. https://doi.org/10.1037/a0030831.

Troy, A. S., Shallcross, A. J., \& Mauss, I. B. (2013). A person-by-situation approach to emotion regulation: Cognitive reappraisal can either help or hurt, depending on the context. Psychological Science, 24, 2505-2514. https://doi.org/10.1177/0956797613496434.

Urry, H. L. (2010). Seeing, thinking, and feeling: Emotion-regulating effects of gaze-directed cognitive reappraisal. Emotion, 10, 125135. https://doi.org/10.1037/a0017434.

Urry, H. L., \& Gross, J. J. (2010). Emotion regulation in older age. Current Directions in Psychological Science, 19, 352-357. https ://doi.org/10.1177/0963721410388395.

van der Does, A. J. W. (2002). De Nederlandse versie van de Beck Depression Inventory - tweede editie [The Dutch version of the Beck depression inventory] (2nd ed.). Lisse: Swets \& Zeitlinger.

Van Reekum, C. M., Johnstone, T., Urry, H. L., Thurow, M. E., Schaefer, H. S., Alexander, A. L., et al. (2007). Gaze fixations predict brain activation during the voluntary regulation of pictureinduced negative affect. NeuroImage, 36, 1041-1055. https://doi. org/10.1016/j.neuroimage.2007.03.052.

Vujovic, L., Opitz, P. C., Birk, J. L., \& Urry, H. L. (2014). Cut! That's a wrap: Regulating negative emotion by ending emotioneliciting situations. Frontiers in Psychology, 5, 165. https://doi. org/10.3389/fpsyg.2014.00165.

Webb, T. L., Miles, E., \& Sheeran, P. (2012a). Dealing with feeling: A meta-analysis of the effectiveness of strategies derived from the process model of emotion regulation. Psychological Bulletin, 138, 775-808. https://doi.org/10.1037/a0027600.

Webb, T. L., Schweiger Gallo, I., Miles, E., Gollwitzer, P. M., \& Sheeran, P. (2012b). Effective regulation of affect: An action control perspective on emotion regulation. European Review of Social Psychology, 23, 143-186. https://doi.org/10.1080/10463 283.2012.718134

Publisher's Note Springer Nature remains neutral with regard to jurisdictional claims in published maps and institutional affiliations. 